\title{
Variational Approach for a Class of Two-point Fractional Boundary Value Systems
}

\author{
${ }^{1}$ Ghasem A. Afrouzi, ${ }^{2}$ Samad Mohseni Kolagar, ${ }^{3}$ Armin Hadjian and ${ }^{4}$ Jiafa $\mathbf{X} \mathbf{u}^{*}$ \\ ${ }^{1,2}$ Department of Mathematics, Faculty of Mathematical Sciences, University of Mazandaran \\ Babolsar, Iran \\ ${ }^{3}$ Department of Mathematics, Faculty of Basic Sciences, University of Bojnord \\ P.O. Box 1339, Bojnord 94531, Iran \\ ${ }^{4}$ School of Mathematical Sciences, Chongqing Normal University \\ Chongqing 401331, China \\ ${ }^{*}$ Corresponding author: xujiafa292@sina.com
}

Article history

Received: 24 November 2016

Received in revised form: 30 March 2018

Accepted: 2 April 2018

Published on line: 1 December 2018

\begin{abstract}
An existence result of multiple solutions for a class of two-point fractional boundary value equations depending upon a positive parameter is established. Our main tool is a three critical points theorem due to Bonanno and Marano [G. Bonanno and S.A. Marano, On the structure of the critical set of non-differentiable functions with a weak compactness condition, Appl. Anal. 89 (2010), 1-10].
\end{abstract}

Keywords two-point fractional boundary value systems; Caputo fractional derivatives; critical point theory; three solutions.

Mathematics Subject Classification 34A08, 26A33, 35A15.

\section{Introduction}

Differential equations with fractional order are generalization of ordinary differential equations to non-integer order. Fractional differential equations have received increasing attention during recent years, since the behavior of physical systems can be properly described by using fractional order system theory. So, fractional differential equations got the attention of many researchers and considerable work has been done in this regard; see the monographs of Miller and Ross [1], Samko et al. [2], Podlubny [3], Hilfer [4], Kilbas et al. [5] and the papers [6-11]. See also [12-16] and references therein.

Recently, fractional differential equations have been of great interest, and boundary value problems for fractional differential equations have been considered by the use of techniques of nonlinear analysis (fixed-point theorems [10,17,18], Leray-Schauder theory [19, 20], lower and upper solution method, and monotone iterative method [21-23]). 
Critical Point theory and variational methods are crucial in the study of many mathematical models of real-world problems. Many applied problems can be understood and solved in terms of the minimization of a functional, usually related to the energy, in an appropriate functional space. Minimization and variational problems are at the interface between nonlinear analysis, calculus of variations, differential equations and mathematical physics and play a fundamental role in the application of mathematics to different scientific areas. The classical critical point theory for $C^{1}$-functional was developed in the sixties and seventies (see [24-27] and references therein). But, to the best of the authors knowledge, there are few results on the solutions to fractional BVP which were established by the critical point theory, since it is often very difficult to establish a suitable space and variational functional for fractional differential equations with boundary conditions. Recently, Jiao and Zhou [28] introduced some appropriate function spaces as their working space.

The aim of this paper is to study the nonlinear fractional boundary value system

$$
\left\{\begin{array}{l}
\frac{d}{d t}\left(\Delta_{\alpha_{i}} u_{i}(t)\right)=\lambda F_{u_{i}}\left(t, u_{1}(t), \ldots, u_{n}(t)\right) \quad \text { a.e. } t \in[0, T] \\
u_{i}(0)=u_{i}(T)=0
\end{array}\right.
$$

where

$$
\Delta_{\alpha_{i}} u_{i}(t):={ }_{0} D_{t}^{\alpha_{i}-1}\left({ }_{0}^{c} D_{t}^{\alpha_{i}} u_{i}(t)\right)-{ }_{t} D_{T}^{\alpha_{i}-1}\left({ }_{t}^{c} D_{T}^{\alpha_{i}} u_{i}(t)\right),
$$

for $1 \leq i \leq n, \alpha_{i} \in(0,1],{ }_{0} D_{t}^{\alpha_{i}-1}$ and ${ }_{t} D_{T}^{\alpha_{i}-1}$ are the left and right Riemann-Liouville fractional integrals of order $1-\alpha$ respectively, ${ }_{0}^{c} D_{t}^{\alpha_{i}}$ and ${ }_{t}^{c} D_{T}^{\alpha_{i}}$ are the left and right Caputo fractional derivatives of order $0<\alpha_{i} \leq 1$ respectively, $\lambda$ is a positive real parameter, $F:[0, T] \times \mathbb{R}^{n} \rightarrow \mathbb{R}$ is a measurable function with respect to $t \in[0, T]$ for every $\left(x_{1}, \ldots, x_{n}\right) \in \mathbb{R}^{n}$ and is $C^{1}$ with respect to $\left(x_{1}, \ldots, x_{n}\right) \in \mathbb{R}^{n}$ for a.e. $t \in[0, T], F_{u_{i}}$ denotes the partial derivative of $F$ with respect to $u_{i}, F(t, 0, \ldots, 0)=0$ for a.e. $t \in[0, T]$, and for every $M>0$ and every $1 \leq i \leq n$,

$$
\sup _{\left|\left(x_{1}, \ldots, x_{n}\right)\right| \leq M}\left|F_{u_{i}}\left(t, x_{1}, \ldots, x_{n}\right)\right| \in L^{1}([0, T]) .
$$

In the present paper, motivated by [29-31], using a three critical points theorem obtained in [32] which we recall in the next section(Theorem 1), we ensure the existence of at least three solutions for system (1). This theorem has been successfully employed to establish the existence of at least three solutions for some boundary value problems (see, e.g., the papers [33-38]).

This paper is organized as follows. In Section 2, we present some necessary preliminary facts that will be needed in the paper. In Section 3 our main result (Theorem 2) and some significative consequences (Corollaries 1 and 2) and an example (Example 1) are presented.

\section{Preliminaries}

In this section, we first introduce some necessary definitions and properties of the fractional calculus which are used in this paper.

Definition $1[5]$ Let $u$ be a function defined on $[a, b]$. The left and right Riemann-Liouville fractional derivatives of order $\alpha>0$ for a function $u$ are defined by

$$
{ }_{a} D_{t}^{\alpha} u(t):=\frac{d^{n}}{d t^{n}} D_{t}^{\alpha-n} u(t)=\frac{1}{\Gamma(n-\alpha)} \frac{d^{n}}{d t^{n}} \int_{a}^{t}(t-s)^{n-\alpha-1} u(s) d s,
$$


and

$$
{ }_{t} D_{b}^{\alpha} u(t):=(-1)^{n} \frac{d^{n}}{d t^{n}}{ }_{t} D_{b}^{\alpha-n} u(t)=\frac{(-1)^{n}}{\Gamma(n-\alpha)} \frac{d^{n}}{d t^{n}} \int_{t}^{b}(t-s)^{n-\alpha-1} u(s) d s,
$$

for every $t \in[a, b]$, provided the right-hand sides are pointwise defined on $[a, b]$, where $n-1 \leq$ $\alpha<n$ and $n \in \mathbb{N}$.

Here, $\Gamma(\alpha)$ is the standard gamma function given by

$$
\Gamma(\alpha):=\int_{0}^{+\infty} z^{\alpha-1} e^{-z} d z
$$

Set $A C^{n}([a, b], \mathbb{R})$ the space of functions $u:[a, b] \rightarrow \mathbb{R}$ such that $u \in C^{n-1}([a, b], \mathbb{R})$ and $u^{(n-1)} \in A C([a, b], \mathbb{R})$. Here, as usual, $C^{n-1}([a, b], \mathbb{R})$ denotes the set of mappings having $(n-1)$ times continuously differentiable on $[a, b]$. In particular, we denote $A C([a, b], \mathbb{R}):=$ $A C^{1}([a, b], \mathbb{R})$.

Definition $2[5]$ Let $\gamma \geq 0$ and $n \in \mathbb{N}$.

(i) If $\gamma \in(n-1, n)$ and $u \in A C^{n}([a, b], \mathbb{R})$, then the left and right Caputo fractional derivatives of order $\gamma$ for function $u$ denoted by ${ }_{a}^{c} D_{t}^{\gamma} u(t)$ and ${ }_{t}^{c} D_{b}^{\gamma} u(t)$, respectively, exist almost everywhere on $[a, b],{ }_{a}^{c} D_{t}^{\gamma} u(t)$ and ${ }_{t}^{c} D_{b}^{\gamma} u(t)$ are represented by

$$
\begin{aligned}
& { }_{a}^{c} D_{t}^{\gamma} u(t):=\frac{1}{\Gamma(n-\gamma)} \int_{a}^{t}(t-s)^{n-\gamma-1} u^{(n)}(s) d s, \\
& { }_{t}^{c} D_{b}^{\gamma} u(t):=\frac{(-1)^{n}}{\Gamma(n-\gamma)} \int_{t}^{b}(s-t)^{n-\gamma-1} u^{(n)}(s) d s,
\end{aligned}
$$

for every $t \in[a, b]$, respectively.

(ii) If $\gamma=n-1$ and $u \in A C^{n-1}([a, b], \mathbb{R})$, then ${ }_{a}^{c} D_{t}^{n-1} u(t)$ and ${ }_{t}^{c} D_{b}^{n-1} u(t)$ are represented by

$$
{ }_{a}^{c} D_{t}^{n-1} u(t)=u^{(n-1)}(t), \quad \text { and } \quad{ }_{t}^{c} D_{b}^{n-1} u(t)=(-1)^{(n-1)} u^{(n-1)}(t),
$$

for every $t \in[a, b]$.

With these definitions, we have the rule for fractional integration by parts, and the composition of the Riemann-Liouville fractional integration operator with the Caputo fractional differentiation operator, which were proved in $[2,5]$.

Proposition $1[2,5]$ We have the following property of fractional integration

$$
\int_{a}^{b}\left[{ }_{a} D_{t}^{-\gamma} u(t)\right] v(t) d t=\int_{a}^{b}\left[{ }_{t} D_{b}^{-\gamma} v(t)\right] u(t) d t, \quad \gamma>0,
$$

provided that $u \in L^{p}([a, b], \mathbb{R}), v \in L^{q}([a, b], \mathbb{R})$ and $p \geq 1, q \geq 1,1 / p+1 / q \leq 1+\gamma$ or $p \neq 1$, $q \neq 1,1 / p+1 / q=1+\gamma$. 
Proposition 2[5] Let $n \in \mathbb{N}$ and $n-1<\gamma \leq n$. If $u \in A C^{n}([a, b], \mathbb{R})$ or $u \in C^{n}([a, b], \mathbb{R})$, then

$$
\begin{gathered}
{ }_{a} D_{t}^{-\gamma}\left({ }_{a}^{c} D_{t}^{\gamma} u(t)\right)=u(t)-\sum_{j=0}^{n-1} \frac{u^{(j)}(a)}{j !}(t-a)^{j}, \\
{ }_{t} D_{b}^{-\gamma}\left({ }_{t}^{c} D_{b}^{\gamma} u(t)\right)=u(t)-\sum_{j=0}^{n-1} \frac{(-1)^{j} u^{(j)}(b)}{j !}(b-t)^{j}
\end{gathered}
$$

for every $t \in[a, b]$. In particular, if $0<\gamma \leq 1$ and $u \in A C([a, b], \mathbb{R})$ or $u \in C^{1}([a, b], \mathbb{R})$, then

$$
{ }_{a} D_{t}^{-\gamma}\left({ }_{a}^{c} D_{t}^{\gamma} u(t)\right)=u(t)-u(a), \quad \text { and }{ }_{t} D_{b}^{-\gamma}\left({ }_{t}^{c} D_{b}^{\gamma} u(t)\right)=u(t)-u(b) .
$$

To establish a variational structure for the main problem, it is necessary to construct appropriate function spaces. Following $[28]$, we denote by $C_{0}^{\infty}([0, T], \mathbb{R})$ the set of all functions $g \in C^{\infty}([0, T], \mathbb{R})$ with $g(0)=g(T)=0$.

Definition 3 [28] Let $0<\alpha_{i} \leq 1$ for $1 \leq i \leq n$. The fractional derivative space $E_{0}^{\alpha_{i}}$ is defined by the closure of $C_{0}^{\infty}([0, T], \mathbb{R})$ with respect to the norm

$$
\left\|u_{i}\right\|:=\left(\int_{0}^{T}\left|{ }_{0}^{c} D_{t}^{\alpha_{i}} u_{i}(t)\right|^{2} d t+\int_{0}^{T}\left|u_{i}(t)\right|^{2} d t\right)^{1 / 2}, \quad \forall u_{i} \in E_{0}^{\alpha_{i}} .
$$

Clearly, the fractional derivative space $E_{0}^{\alpha_{i}}$ is the space of functions $u_{i} \in L^{2}([0, T], \mathbb{R})$ having an $\alpha_{i}$-order Caputo fractional derivative ${ }_{0}^{c} D_{t}^{\alpha_{i}} u_{i} \in L^{2}([0, T], \mathbb{R})$ and $u_{i}(0)=u_{i}(T)=0$ for $1 \leq i \leq n$. From [28, Proposition 3.1], we know for $0<\alpha_{i} \leq 1$, the space $E_{0}^{\alpha_{i}}$ is a reflexive and separable Banach space.

For every $u_{i} \in E_{0}^{\alpha_{i}}$, set

$$
\left\|u_{i}\right\|_{L^{s}}:=\left(\int_{0}^{T}\left|u_{i}(t)\right|^{s} d t\right)^{1 / s}, \quad s \geq 1,
$$

and

$$
\left\|u_{i}\right\|_{\infty}:=\max _{t \in[0, T]}\left|u_{i}(t)\right| .
$$

Lemma $1[28]$ Let $\alpha_{i} \in(1 / 2,1]$ for $1 \leq i \leq n$. For all $u_{i} \in E_{0}^{\alpha_{i}}$, we have

$$
\begin{gathered}
\left\|u_{i}\right\|_{L^{2}} \leq \frac{T^{\alpha_{i}}}{\Gamma\left(\alpha_{i}+1\right)}\left\|_{0}^{c} D_{t}^{\alpha_{i}} u_{i}\right\|_{L^{2}}, \\
\left\|u_{i}\right\|_{\infty} \leq \frac{T^{\alpha_{i}-1 / 2}}{\Gamma\left(\alpha_{i}\right) \sqrt{\left(2 \alpha_{i}-1\right)}}\left\|_{0}^{c} D_{t}^{\alpha_{i}} u_{i}\right\|_{L^{2}} .
\end{gathered}
$$

According to (5), we can consider $E_{0}^{\alpha_{i}}$ with respect to the norm

$$
\left\|u_{i}\right\|_{\alpha_{i}}:=\left(\left.\left.\int_{0}^{T}\right|_{0} ^{c} D_{t}^{\alpha_{i}} u_{i}(t)\right|^{2} d t\right)^{1 / 2}=\left\|_{0}^{c} D_{t}^{\alpha_{i}} u_{i}\right\|_{L^{2}}, \quad \forall u_{i} \in E_{0}^{\alpha_{i}}
$$

for $1 \leq i \leq n$, which is equivalent to (3). 
Lemma $2[28]$ Let $\alpha_{i} \in(1 / 2,1]$ for $1 \leq i \leq n$, then for every $u_{i} \in E_{0}^{\alpha_{i}}$, we have

$$
\left|\cos \left(\pi \alpha_{i}\right)\right|\left\|u_{i}\right\|_{\alpha_{i}}^{2} \leq-\int_{0}^{T}{ }_{0}^{c} D_{t}^{\alpha_{i}} u_{i}(t) \cdot{ }_{t}^{c} D_{T}^{\alpha_{i}} u_{i}(t) d t \leq \frac{1}{\left|\cos \left(\pi \alpha_{i}\right)\right|}\left\|u_{i}\right\|_{\alpha_{i}}^{2} .
$$

Throughout this paper, we let $X$ be the Cartesian product of the $n$ spaces $E_{0}^{\alpha_{i}}$ for $1 \leq i \leq n$, i.e., $X=E_{0}^{\alpha_{1}} \times E_{0}^{\alpha_{2}} \times \cdots \times E_{0}^{\alpha_{n}}$ equipped with the norm

$$
\|u\|:=\sum_{i=1}^{n}\left\|u_{i}\right\|_{\alpha_{i}}, \quad u=\left(u_{1}, u_{2}, \ldots, u_{n}\right)
$$

where $\left\|u_{i}\right\|_{\alpha_{i}}$ is defined in (7). Obviously, $X$ is compactly embedded in $(C([0, T], \mathbb{R}))^{n}$.

We mean by a (weak) solution of system (1), any $u=\left(u_{1}, u_{2}, \ldots, u_{n}\right) \in X$ such that

$$
\begin{gathered}
\int_{0}^{T} \sum_{i=1}^{n}\left({ }_{0} D_{t}^{\alpha_{i}-1}\left({ }_{0}^{c} D_{t}^{\alpha_{i}} u_{i}(t)\right)-{ }_{t} D_{T}^{\alpha_{i}-1}\left({ }_{t}^{c} D_{T}^{\alpha_{i}} u_{i}(t)\right)\right) \cdot v_{i}^{\prime}(t) d t \\
\quad-\lambda \int_{0}^{T} \sum_{i=1}^{n} F_{u_{i}}\left(t, u_{1}(t), \ldots, u_{n}(t)\right) v_{i}(t) d t=0
\end{gathered}
$$

for all $v=\left(v_{1}, v_{2}, \ldots, v_{n}\right) \in X$.

Here we recall for the reader's convenience the three critical points theorem of [32] which is our main tool to prove the results. Here, $X^{*}$ denotes the dual space of $X$.

Theorem 1 Let $X$ be a reflexive real Banach space, $\Phi: X \rightarrow \mathbb{R}$ be a coercive continuously Gâteaux differentiable and sequentially weakly lower semicontinuous functional whose Gâteaux derivative admits a continuous inverse on $X^{*}, \Psi: X \rightarrow \mathbb{R}$ be a continuously Gâteaux differentiable functional whose Gâteaux derivative is compact such that

$$
\Phi(0)=\Psi(0)=0
$$

Assume that there exists $r>0$ and $\bar{x} \in X$, with $r<\Phi(\bar{x})$, such that

(i) $\frac{\sup _{\Phi(x) \leq r} \Psi(x)}{r}<\frac{\Psi(\bar{x})}{\Phi(\bar{x})}$;

(ii) for each $\left.\lambda \in \Lambda_{r}:=\right] \frac{\Phi(\bar{x})}{\Psi(\bar{x})}, \frac{r}{\sup _{\Phi(x) \leq r} \Psi(x)}[$, the functional $\Phi-\lambda \Psi$ is coercive.

Then, for each $\lambda \in \Lambda_{r}$ the functional $\Phi-\lambda \Psi$ has at least three distinct critical points in $X$.

\section{Main Results}

In the present section we discuss the existence of multiple solutions for system (1). For any $\gamma>0$, we denote by $K(\gamma)$ the set

$$
\left\{\left(x_{1}, \ldots, x_{n}\right) \in \mathbb{R}^{n}: \sum_{i=1}^{n}\left|\cos \left(\pi \alpha_{i}\right)\right| \cdot\left|x_{i}\right|^{2} \leq \gamma\right\} .
$$


This set will be used in some of our hypotheses with appropriate choices of $\gamma$.

Moreover, put

$$
c:=\max \left\{\frac{T^{2 \alpha_{i}-1}}{\left(\Gamma\left(\alpha_{i}\right)\right)^{2}\left(2 \alpha_{i}-1\right)}: \text { for } 1 \leq i \leq n\right\}
$$

Theorem 2 Let $\frac{1}{2}<\alpha_{i} \leq 1$ for $1 \leq i \leq n$. Furthermore, assume that there exist a positive constant $r$ and a function $w=\left(w_{1}, \ldots, w_{n}\right) \in X$ such that

(i) $-\int_{0}^{T} \sum_{i=1}^{n}{ }_{0}^{c} D_{t}^{\alpha_{i}} w_{i}(t) \cdot{ }_{t}^{c} D_{T}^{\alpha_{i}} w_{i}(t) d t>r$;

(ii) $r \frac{\int_{0}^{T} F\left(t, w_{1}, \ldots, w_{n}\right) d t}{\sum_{i=1}^{n} \frac{\left\|w_{i}\right\|_{\alpha_{i}}^{2}}{\left|\cos \left(\pi \alpha_{i}\right)\right|}}-\int_{0}^{T} \max _{\left(x_{1}, \ldots, x_{n}\right) \in K(c r)} F\left(t, x_{1}, \ldots, x_{n}\right) d t>0$;

(iii) $\limsup _{\begin{array}{c}\left(\left|x_{1}\right|, \ldots,\left|x_{n}\right|\right) \rightarrow(+\infty, \ldots,+\infty) \\ \text { with respect to } t \in[0, T] .\end{array}} \frac{F\left(t, x_{1}, \ldots, x_{n}\right)}{\sum_{i=1}^{n}\left|\cos \left(\pi \alpha_{i}\right)\right| \cdot\left|x_{i}\right|^{2}}<\frac{\int_{0}^{T} \max _{\left(x_{1}, \ldots, x_{n}\right) \in K(c r)} F\left(t, x_{1}, \ldots, x_{n}\right) d t}{c r T}$ uniformly

Then, for each

$$
\lambda \in \Lambda:=] \frac{\sum_{i=1}^{n} \frac{\left\|w_{i}\right\|_{\alpha_{i}}^{2}}{\left|\cos \left(\pi \alpha_{i}\right)\right|}}{\int_{0}^{T} F\left(t, w_{1}(t), \ldots, w_{n}(t)\right) d t}, \frac{r}{\int_{0}^{T} \max _{\left(x_{1}, \ldots, x_{n}\right) \in K(c r)} F\left(t, x_{1}, \ldots, x_{n}\right) d t}[
$$

system (1) admits at least three solutions in $X$.

Proof For each $u=\left(u_{1}, \ldots, u_{n}\right) \in X$, define $\Phi, \Psi: X \rightarrow \mathbb{R}$ as

$$
\Phi(u):=-\int_{0}^{T} \sum_{i=1}^{n}{ }_{0}^{c} D_{t}^{\alpha_{i}} u_{i}(t) \cdot{ }_{t}^{c} D_{T}^{\alpha_{i}} u_{i}(t) d t
$$

and

$$
\Psi(u):=\int_{0}^{T} F\left(t, u_{1}(t), \ldots, u_{n}(t)\right) d t .
$$

Clearly, $\Phi$ and $\Psi$ are continuously Gâteaux differentiable functionals whose Gâteaux derivatives at the point $u=\left(u_{1}, \ldots, u_{n}\right) \in X$ are given by

$$
\begin{gathered}
\Phi^{\prime}(u)(v)=-\int_{0}^{T} \sum_{i=1}^{n}\left({ }_{0}^{c} D_{t}^{\alpha_{i}} u_{i}(t) \cdot{ }_{t}^{c} D_{T}^{\alpha_{i}} v_{i}(t)+{ }_{t}^{c} D_{T}^{\alpha_{i}} u_{i}(t) \cdot{ }_{0}^{c} D_{t}^{\alpha_{i}} v_{i}(t)\right) d t, \\
\Psi^{\prime}(u)(v)=\int_{0}^{T} \sum_{i=1}^{n}\left(F_{u_{i}}\left(x, u_{1}(x), \ldots, u_{n}(x)\right) v_{i}(x) d x,\right.
\end{gathered}
$$

for every $v=\left(v_{1}, \ldots, v_{n}\right) \in X$. By Definition 2 and (2), we have

$$
\Phi^{\prime}(u)(v)=\int_{0}^{T} \sum_{i=1}^{n}\left({ }_{0} D_{t}^{\alpha_{i}-1}\left({ }_{0}^{c} D_{t}^{\alpha_{i}} u_{i}(t)\right)-{ }_{t} D_{T}^{\alpha_{i}-1}\left({ }_{t}^{c} D_{T}^{\alpha_{i}} u_{i}(t)\right)\right) \cdot v_{i}^{\prime}(t) d t .
$$


Hence, $\Phi-\lambda \Psi \in C^{1}(X, \mathbb{R})$. Moreover, $\Psi^{\prime}: X \rightarrow X^{*}$ is a compact operator (see the proof of Theorem 3.1 in [31]). Furthermore, similar to the proof of [39, Theorem 3.1], we can show that $\Phi$ is sequentially weakly lower semicontinuous. Also, $\Phi^{\prime}: X \rightarrow X^{*}$ admits a continuous inverse on $X^{*}$. Further, from Lemma 2, the functional $\Phi$ is coercive. Indeed, one has

$$
\Phi(u) \geq \sum_{i=1}^{n}\left|\cos \left(\pi \alpha_{i}\right)\right|\left\|u_{i}\right\|_{\alpha_{i}}^{2} \rightarrow+\infty,
$$

as $\|u\| \rightarrow+\infty$. Moreover, we have $\Phi(0)=\Psi(0)=0$.

Clearly, the required hypothesis $\Phi(\bar{x})>r$ follows from (i) and the definition of $\Phi$ by choosing $\bar{x}=w$. From (4) and (6), for every $u_{i} \in E_{0}^{\alpha_{i}}$ we have

$$
\max _{t \in[0, T]}\left|u_{i}(t)\right|^{2} \leq c\left\|u_{i}\right\|_{\alpha_{i}}^{2}
$$

for $1 \leq i \leq n$. Hence

$$
\max _{t \in[0, T]} \sum_{i=1}^{n}\left|u_{i}(t)\right|^{2} \leq c \sum_{i=1}^{n}\left\|u_{i}\right\|_{\alpha_{i}}^{2},
$$

for each $u=\left(u_{1}, u_{2}, \ldots, u_{n}\right) \in X$. From Lemma 2, (6) and (8), for each $r>0$ we obtain

$$
\begin{aligned}
\Phi^{-1}((-\infty, r]) & =\left\{u=\left(u_{1}, \ldots, u_{n}\right) \in X: \Phi(u) \leq r\right\} \\
& \subseteq\left\{u=\left(u_{1}, \ldots, u_{n}\right) \in X: \sum_{i=1}^{n}\left|\cos \left(\pi \alpha_{i}\right)\right|\left\|u_{i}\right\|_{\alpha_{i}}^{2} \leq r\right\} \\
& \subseteq\left\{u=\left(u_{1}, \ldots, u_{n}\right) \in X: \sum_{i=1}^{n}\left|\cos \left(\pi \alpha_{i}\right)\right| \frac{\left(\Gamma\left(\alpha_{i}\right)\right)^{2}\left(2 \alpha_{i}-1\right)}{T^{2 \alpha_{i}-1}}\left\|u_{i}\right\|_{\infty}^{2} \leq r\right\} \\
& \subseteq\left\{u=\left(u_{1}, \ldots, u_{n}\right) \in X: \sum_{i=1}^{n}\left|\cos \left(\pi \alpha_{i}\right)\right| \cdot\left|u_{i}(t)\right|^{2} \leq c r, \text { for all } t \in[0, T]\right\} .
\end{aligned}
$$

Then,

$$
\begin{aligned}
\sup _{u \in \Phi^{-1}((-\infty, r])} \Psi(u) & =\sup _{u \in \Phi^{-1}((-\infty, r])} \int_{0}^{T} F\left(t, u_{1}, \ldots, u_{n}\right) d t \\
& \leq \int_{0}^{T} \max _{\left(x_{1}, \ldots, x_{n}\right) \in K(c r)} F\left(t, x_{1}, \ldots, x_{n}\right) d t .
\end{aligned}
$$

Therefore, from the condition (ii), we have

$$
\begin{aligned}
\sup _{u \in \Phi^{-1}((-\infty, r])} \Psi(u) & \leq \int_{0}^{T} \max _{\left(x_{1}, \ldots, x_{n}\right) \in K(c r)} F\left(t, x_{1}, \ldots, x_{n}\right) d t \\
& <r \frac{\int_{0}^{T} F\left(t, w_{1}, \ldots, w_{n}\right) d t}{\sum_{i=1}^{n} \frac{\left\|w_{i}\right\|_{\alpha_{i}}^{2}}{\left|\cos \left(\pi \alpha_{i}\right)\right|}} \\
& \leq r \frac{\int_{0}^{T} F\left(t, w_{1}, \ldots, w_{n}\right) d t}{\Phi(w)} \\
& =r \frac{\Psi(w)}{\Phi(w)}
\end{aligned}
$$


from which $\left(\mathrm{a}_{1}\right)$ of Theorem 1 follows. Now, fixed $\lambda \in \Lambda$, due to (iii), we can find $\gamma, \vartheta \in \mathbb{R}$ with

$$
0<\gamma<\frac{\int_{0}^{T} \max _{\left(x_{1}, \ldots, x_{n}\right) \in K(c r)} F\left(t, x_{1}, \ldots, x_{n}\right) d t}{r}
$$

such that

$$
c T F\left(t, x_{1}, \ldots, x_{n}\right) \leq \gamma \sum_{i=1}^{n}\left|x_{i}\right|^{2}\left|\cos \left(\pi \alpha_{i}\right)\right|+\vartheta,
$$

for all $t \in[0, T]$ and for all $\left(x_{1}, \ldots, x_{n}\right) \in \mathbb{R}^{n}$. Fixed $\left(u_{1}, \ldots, u_{n}\right) \in X$, bearing in mind (8), we have

$$
\begin{aligned}
\Phi(u)-\lambda \Psi(u) & \geq \sum_{i=1}^{n}\left|\cos \left(\pi \alpha_{i}\right)\right|\left\|u_{i}\right\|_{\alpha_{i}}^{2}-\lambda \int_{0}^{T} F\left(t, u_{1}, \ldots, u_{n}\right) d t \\
& \geq \sum_{i=1}^{n}\left|\cos \left(\pi \alpha_{i}\right)\right|\left\|u_{i}\right\|_{\alpha_{i}}^{2}-\frac{\lambda \gamma}{c T} \sum_{i=1}^{n} \int_{0}^{T}\left|\cos \left(\pi \alpha_{i}\right)\right| \cdot\left|u_{i}(t)\right|^{2} d t-\frac{\lambda \vartheta}{c} \\
& \geq \sum_{i=1}^{n}\left|\cos \left(\pi \alpha_{i}\right)\right|\left\|u_{i}\right\|_{\alpha_{i}}^{2}-\lambda \gamma \sum_{i=1}^{n}\left|\cos \left(\pi \alpha_{i}\right)\right|\left\|u_{i}\right\|_{\alpha_{i}}^{2}-\frac{\lambda \vartheta}{c} \\
& \geq\left(1-\frac{\gamma r}{\int_{0}^{T} \max _{\left(x_{1}, \ldots, x_{n}\right) \in K(c r)} F\left(t, x_{1}, \ldots, x_{n}\right) d t}\right) \sum_{i=1}^{n}\left|\cos \left(\pi \alpha_{i}\right)\right|\left\|u_{i}\right\|_{\alpha_{i}}^{2}-\frac{\lambda \vartheta}{c} .
\end{aligned}
$$

Thus

$$
\lim _{\|u\| \rightarrow+\infty}(\Phi(u)-\lambda \Psi(u))=+\infty
$$

i.e., $\Phi-\lambda \Psi$ is coercive. Now, all the hypotheses of Theorem 1 are satisfied. Also note that the solutions of the equation $\Phi^{\prime}(u)-\lambda \Psi^{\prime}(u)=0$ are exactly the solutions of (1) (see [40]). Thus, for each $\lambda \in \Lambda$, system (1) admits at least three solutions in $X$.

Example 1 Consider the system

$$
\left\{\begin{array}{l}
\frac{d}{d t}\left(\Delta_{0.7} u_{1}(t)\right)=\lambda F_{u_{1}}\left(t, u_{1}(t), u_{2}(t)\right) \quad \text { a.e. } t \in[0,1] \\
\frac{d}{d t}\left(\Delta_{0.75} u_{2}(t)\right)=\lambda F_{u_{2}}\left(t, u_{1}(t), u_{2}(t)\right) \\
u_{1}(0)=u_{2}(0)=u_{1}(1)=u_{2}(1)=0 .
\end{array}\right.
$$

For all $\left(t, x_{1}, x_{2}\right) \in[0,1] \times \mathbb{R}^{2}$, put

$$
F\left(t, x_{1}, x_{2}\right)=t^{3} \begin{cases}-\left(\cos (0.7 \pi) x_{1}^{2}+\cos (0.75 \pi) x_{2}^{2}\right)^{2} & \\ +2\left(\cos (0.7 \pi) x_{1}^{2}+\cos (0.75 \pi) x_{2}^{2}\right) & \cos (0.7 \pi) x_{1}^{2}+\cos (0.75 \pi) x_{2}^{2} \leq 1 \\ 1 & \cos (0.7 \pi) x_{1}^{2}+\cos (0.75 \pi) x_{2}^{2}>1\end{cases}
$$

Clearly, $F(t, 0,0)=0$ for all $t \in[0,1]$. With the aid of direct computation we have that $c \approx 1.4837$. By choosing, for instance, $w_{1}(t)=\frac{1}{2}\left(t^{5}-t^{10}\right), w_{2}(t)=3\left(t^{5}-t^{10}\right), r=\frac{1}{10^{4}}$, and by a simple calculation, we obtain that

$$
\left\|w_{1}\right\|_{0.7}^{2} \approx 0.0016, \quad\left\|w_{2}\right\|_{0.75}^{2} \approx 0.0395
$$


The conditions (i), (ii) and (iii) of Theorem 2 are satisfied. In fact,

$$
\begin{aligned}
-\int_{0}^{T} \sum_{i=1}^{2}{ }_{0}^{c} D_{t}^{\alpha_{i}} w_{i}(t) \cdot{ }_{t}^{c} D_{T}^{\alpha_{i}} w_{i}(t) d t & \geq|\cos (0.7 \pi)|\left\|w_{1}\right\|_{0.7}^{2}+|\cos (0.75 \pi)|\left\|w_{2}\right\|_{0.75}^{2} \\
& \approx 0.0009+0.0279 \approx 0.0288>\frac{1}{10^{4}}=r
\end{aligned}
$$

and

$$
\begin{aligned}
\frac{\int_{0}^{1} \max _{\left(x_{1}, x_{2}\right) \in K(c r)} F\left(t, x_{1}, x_{2}\right) d t}{r}=\frac{c}{2} & \approx 0.7418 \\
& <\frac{\int_{0}^{1} F\left(t, w_{1}, w_{2}\right) d t}{\sum_{i=1}^{2} \frac{\left\|w_{i}\right\|_{\alpha_{i}}^{2}}{\cos \left(\pi \alpha_{i}\right) \mid}} \\
& \approx 17.0940 .
\end{aligned}
$$

Note that

$$
\limsup _{\left(\left|x_{1}\right|,\left|x_{2}\right|\right) \rightarrow(+\infty,+\infty)} \frac{F\left(t, x_{1}, x_{2}\right)}{\sum_{i=1}^{2}\left|\cos \left(\pi \alpha_{i}\right)\right| \cdot\left|x_{i}\right|^{2}}=0<\frac{\int_{0}^{1} \max _{\left(x_{1}, x_{2}\right) \in K(c r)} F\left(t, x_{1}, x_{2}\right) d t}{c r T}=\frac{1}{2},
$$

uniformly with respect to $t \in[0,1]$. Hence, Theorem 2 is applicable to system (9) for

$$
\lambda \in[0.0585,1.3480] .
$$

Next, we want to give a verifiable consequence of Theorem 2 for a fixed text function $w$. For all $1 \leq i \leq n$, set

$$
\begin{aligned}
& P_{\alpha_{i}}:= \frac{16}{T^{2} \Gamma^{2}\left(2-\alpha_{i}\right)}\left\{\int_{0}^{\frac{T}{4}} t^{2-2 \alpha_{i}} d t+\int_{\frac{T}{4}}^{\frac{3 T}{4}}\left[t^{1-\alpha_{i}}-\left(t-\frac{T}{4}\right)^{1-\alpha_{i}}\right]^{2} d t\right. \\
&\left.+\int_{\frac{3 T}{4}}^{T}\left[t^{1-\alpha_{i}}-\left(t-\frac{T}{4}\right)^{1-\alpha_{i}}+\left(t-\frac{3 T}{4}\right)^{1-\alpha_{i}}\right]^{2} d t\right\}, \\
& \Delta:=\min \left\{\left|\cos \left(\pi \alpha_{i}\right)\right| P_{\alpha_{i}}: \text { for } 1 \leq i \leq n\right\}, \\
& \Delta^{\prime}:=\max \left\{\frac{P_{\alpha_{i}}}{\left|\cos \left(\pi \alpha_{i}\right)\right|}: \text { for } 1 \leq i \leq n\right\} .
\end{aligned}
$$

Corollary 1 Assume that there exist positive constants $h$ and $d$ such that $\frac{h}{\Delta c n}<d^{2}$, and

(i) $F\left(t, x_{1}, \ldots, x_{n}\right) \geq 0$, for each $\left(t, x_{1}, \ldots, x_{n}\right) \in\left(\left[0, \frac{T}{4}\right] \cup\left[\frac{3 T}{4}, T\right]\right) \times([0, d])^{n}$;

$$
\frac{\int_{0}^{T} \max _{\left(x_{1}, \ldots, x_{n}\right) \in K(h)} F\left(t, x_{1}, \ldots, x_{n}\right) d t}{h}<\frac{\int_{\frac{T}{4}}^{\frac{3 T}{4}} F(t, d, \ldots, d) d t}{n c \Delta^{\prime} d^{2}}
$$


(iii)

$$
\limsup _{\left(\left|x_{1}\right|, \ldots,\left|x_{n}\right|\right) \rightarrow(+\infty, \ldots,+\infty)} \frac{\sup _{t \in[0, T]} F\left(t, x_{1}, \ldots, x_{n}\right)}{\sum_{i=1}^{n}\left|\cos \left(\pi \alpha_{i}\right)\right| \cdot\left|x_{i}\right|^{2}} \leq 0 .
$$

Then, for each

$$
\lambda \in] \frac{n \Delta^{\prime} d^{2}}{\int_{\frac{T}{4}}^{\frac{3 T}{4}} F(t, d, \ldots, d) d t}, \frac{h}{c \int_{0}^{T} \max _{\left(x_{1}, \ldots, x_{n}\right) \in K(h)} F\left(t, x_{1}, \ldots, x_{n}\right) d t}[,
$$

system (1) admits at least three solutions in $X$.

Proof For $d>0$, choose $w(t)=\left(w_{1}(t), \ldots, w_{n}(t)\right)$ for every $t \in[0, T]$ with

$$
w_{i}(t):=\left\{\begin{array}{l}
\frac{4 d}{T} t, \quad t \in\left[0, \frac{T}{4}\right), \\
d, \quad t \in\left[\frac{T}{4}, \frac{3 T}{4}\right] \\
\frac{4 d}{T}(T-t), \quad t \in\left(\frac{3 T}{4}, T\right],
\end{array}\right.
$$

for $1 \leq i \leq n$. Clearly $w_{i}(0)=w_{i}(T)=0$ and $w_{i} \in L^{2}([0, T], \mathbb{R})$ for $1 \leq i \leq n$. A direct calculation shows that

$$
{ }_{0}^{c} D_{t}^{\alpha_{i}} w_{i}(t)=\left\{\begin{array}{l}
\frac{4 d}{T \Gamma\left(2-\alpha_{i}\right)} t^{1-\alpha_{i}}, \quad t \in\left[0, \frac{T}{4}\right), \\
\frac{4 d}{T \Gamma\left(2-\alpha_{i}\right)}\left(t^{1-\alpha_{i}}-\left(t-\frac{T}{4}\right)^{1-\alpha_{i}}\right), \quad t \in\left[\frac{T}{4}, \frac{3 T}{4}\right], \\
\frac{4 d}{T \Gamma\left(2-\alpha_{i}\right)}\left(t^{1-\alpha_{i}}-\left(t-\frac{T}{4}\right)^{1-\alpha_{i}}+\left(t-\frac{3 T}{4}\right)^{1-\alpha_{i}}\right), \quad t \in\left(\frac{3 T}{4}, T\right],
\end{array}\right.
$$

for $1 \leq i \leq n$. Furthermore, ${ }_{0}^{c} D_{t}^{\alpha_{i}} w_{i}$ is continuous on $[0, T]$ and

$$
\begin{aligned}
\left.\int_{0}^{T}{ }_{0}^{c} D_{t}^{\alpha_{i}} w_{i}(t)\right|^{2} d t= & \frac{16 d^{2}}{T^{2} \Gamma^{2}\left(2-\alpha_{i}\right)}\left\{\int_{0}^{\frac{T}{4}} t^{2-2 \alpha_{i}} d t+\int_{\frac{T}{4}}^{\frac{3 T}{4}}\left[t^{1-\alpha_{i}}-\left(t-\frac{T}{4}\right)^{1-\alpha_{i}}\right]^{2} d t\right. \\
& \left.+\int_{\frac{3 T}{4}}^{T}\left[t^{1-\alpha_{i}}-\left(t-\frac{T}{4}\right)^{1-\alpha_{i}}+\left(t-\frac{3 T}{4}\right)^{1-\alpha_{i}}\right]^{2} d t\right\} \\
& =P_{\alpha_{i}} d^{2}
\end{aligned}
$$

for $1 \leq i \leq n$. Thus, $w \in X$, and

$$
\left\|w_{i}\right\|_{\alpha_{i}}^{2}=\int_{0}^{T}\left|{ }_{0}^{c} D_{t}^{\alpha_{i}} w_{i}(t)\right|^{2} d t=P_{\alpha_{i}} d^{2}
$$

for $1 \leq i \leq n$. This and Lemma 2 imply that

$$
\begin{aligned}
\Phi(w)=\Phi\left(w_{1}, \ldots, w_{n}\right) & \geq \sum_{i=1}^{n}\left|\cos \left(\pi w_{i}\right)\right|\left\|w_{i}\right\|_{\alpha_{i}}^{2} \\
& =\sum_{i=1}^{n}\left|\cos \left(\pi \alpha_{i}\right)\right| P_{\alpha_{i}} d^{2} \\
& \geq n \Delta d^{2} .
\end{aligned}
$$


Similarly to (10) we have $\Phi(w) \leq n \Delta^{\prime} d^{2}$.

Let $r=\frac{h}{c}$. From $\frac{h}{\Delta c n}<d^{2}$ we have

$$
\Phi(w) \geq n \Delta d^{2}>n \Delta \times \frac{h}{\Delta c n}=r,
$$

which is (i) of Theorem 2.

On the other hand, by using assumption (j), we infer

$$
\begin{aligned}
\Psi(w) & =\int_{0}^{T} F\left(t, w_{1}(t), \ldots, w_{n}(t)\right) d t \\
& \geq \int_{\frac{T}{4}}^{\frac{3 T}{4}} F(t, d, \ldots, d) d t .
\end{aligned}
$$

Moreover, by condition (jj) we have

$$
\begin{aligned}
\frac{\int_{0}^{T} \max _{\left(x_{1}, \ldots, x_{n}\right) \in K(c r)} F\left(t, x_{1}, \ldots, x_{n}\right) d t}{r} & =\frac{c \int_{0}^{T} \max _{\left(x_{1}, \ldots, x_{n}\right) \in K(h)} F\left(t, x_{1}, \ldots, x_{n}\right) d t}{h} \\
& <\frac{\int_{\frac{T}{4}}^{\frac{3 T}{4}} F(t, d, \ldots, d) d t}{n \Delta^{\prime} d^{2}} \\
& \leq \frac{\left.\int_{0}^{T} F\left(t, w_{1}, \ldots, w_{n}\right)\right) d t}{\sum_{i=1}^{n} \frac{\left\|w_{i}\right\|_{\alpha_{i}}^{2}}{\left|\cos \left(\pi \alpha_{i}\right)\right|}}
\end{aligned}
$$

which implies that (ii) is satisfied. Finally (jjj) implies (iii). Thus, all the assumptions of Theorem 2 are satisfied and the proof is complete.

We now point out the following special case of 1 when $F$ does not depend on $t \in[0, T]$.

Corollary 2 Let $F: \mathbb{R}^{n} \rightarrow \mathbb{R}$ be a $\mathrm{C}^{1}$-function, $F(0, \ldots, 0)=0$ and assume that there exist positive constants $h$ and $d$ such that $\frac{h}{\Delta c n}<d^{2}$, and

(i) $F\left(x_{1}, \ldots, x_{n}\right) \geq 0$, for each $\left(x_{1}, \ldots, x_{n}\right) \in([0, d])^{n}$;

(ii)

$$
\begin{aligned}
& \text { (ii) } \frac{\max _{\left(x_{1}, \ldots, x_{n}\right) \in K(h)} F\left(x_{1}, \ldots, x_{n}\right)}{h}<\frac{F(d, \ldots, d)}{2 n c \Delta^{\prime} d^{2}} ; \\
& \text { (iii) } \limsup _{\left(\left|x_{1}\right|, \ldots,\left|x_{n}\right|\right) \rightarrow(+\infty, \ldots,+\infty)} \frac{F\left(x_{1}, \ldots, x_{n}\right)}{\sum_{i=1}^{n}\left|\cos \left(\pi \alpha_{i}\right)\right| \cdot\left|x_{i}\right|^{2}} \leq 0 .
\end{aligned}
$$

Then, for each

$$
\lambda \in] \frac{2 n \Delta^{\prime} d^{2}}{T F(d, \ldots, d)}, \frac{h}{c T \max _{\left(x_{1}, \ldots, x_{n}\right) \in K(h)} F\left(x_{1}, \ldots, x_{n}\right)}[,
$$

the system

$$
\left\{\begin{array}{l}
\frac{d}{d t}\left(\Delta_{\alpha_{i}} u_{i}(t)\right)=\lambda F_{u_{i}}\left(u_{1}(t), \ldots, u_{n}(t)\right) \quad \text { a.e. } t \in[0, T], \\
u_{i}(0)=u_{i}(T)=0,
\end{array}\right.
$$

admits at least three solutions in $X$. 
Proof The proof is similar to Corollary 1.

\section{Conclusion}

Based on a recent three critical points theorem obtained by Bonanno and Marano [32], we established the existence of an open interval $] \lambda^{\prime}, \lambda^{\prime \prime}[$ for each $\lambda$ in the interval a class of two-point fractional boundary value equations depending on $\lambda$ admits at least three solutions. Also, an example was presented to illustrate the results.

Acknowledge Research supported by the National Natural Science Foundation of China (11601048), Natural Science Foundation of Chongqing (cstc2016jcyjA0181), the Science and Technology Research Program of Chongqing Municipal Education Commission (KJ1703050), Natural Science Foundation of Chongqing Normal University (16XYY24).

\section{References}

[1] Miller, K.S. and Ross, B. An Introduction to the Fractional Calculus and Fractional Differential Equations. New York: Wiley . 1993.

[2] Samko, S.G. Kilbas, A. A. and Marichev, O.I. Fractional Integral and Derivatives: Theory and Applications. Longhorne, PA: Gordon and Breach. 1993.

[3] Podlubny, I. Fractional Differential Equations. San Diego: Academic Press. 1999.

[4] Hilfer, R. Applications of Fractional Calculus in Physics. Singapore: World Scientific. 2000.

[5] Kilbas, A. A. Srivastava, H. M. and Trujillo, J. J. Theory and Applications of Fractional Differential Equations. Amsterdam: Elsevier. 2006.

[6] Agarwal, R.P. Benchohra M. and Hamani, S. A survey on existence results for boundary value problems of nonlinear fractional differential equations and inclusions. Acta Appl. Math. 2010. 109 (3): 973-1033.

[7] Hao, X. Positive solution for singular fractional differential equations involving derivatives. Advances in Difference Equations. 2016. Article ID 139, 12 pages.

[8] Guan, Y. Zhao, Z. and Lin, X. On the existence of positive solutions and negative solutions of singular fractional differential equations via global bifurcation techniques. Boundary Value Problems. 2016. Article ID 141, 18 pages.

[9] Bai, C. Solvability of multi-point boundary value problem of nonlinear impulsive fractional differential equation at resonance. Electron. J. Qual. Theory Differ. Equ. 2011. 2011(89): $1-19$.

[10] Bai, Z. and Sun, W. Existence and multiplicity of positive solutions for singular fractional boundary value problems. Comput. Math. Appl. 2012. 63(9): 1369-1381. 
[11] $\mathrm{Xu}, \mathrm{R}$. and Zhang, Y. Generalized Gronwall fractional summation inequalities and their applications. Journal of Inequalities and Applications. 2015. Article ID 242, 10 pages.

[12] Bai, Z. and Zhang, Y. Solvability of fractional three-point boundary value problems with nonlinear growth. Appl. Math. Comput. 2011. 218 (5): 1719-1725.

[13] Lakshmikantham V. and Vatsala, A.S. Basic theory of fractional differential equations. Nonlinear Anal. 2008. 69 (8): 2677-2682.

[14] Zhang, K. On a sign-changing solution for some fractional differential equations. Boundary Value Problems. 2017. Article ID 59, 8 pages.

[15] Benchohra, M. Hamani, S. and Ntouyas, S.K. Boundary value problems for differential equations with fractional order and nonlocal conditions. Nonlinear Anal. 2009. 71 (7-8): 2391-2396.

[16] Agarwal, R.P. O'Regan, D. and Staněk, S. Positive solutions for Dirichlet problems of singular nonlinear fractional differential equations. J. Math. Anal. Appl. 2010. 371 (1): $57-68$.

[17] Zuo, M. Hao, X. Liu, L. and Cui, Y. Existence results for impulsive fractional integrodifferential equation of mixed type with constant coefficient and antiperiodic boundary conditions. Boundary Value Problems. 2017. Article ID 161, 15 pages.

[18] Jiang, J. Liu, L. and Wu, Y. Positive solutions to singular fractional differential system with coupled boundary conditions. Commun. Nonlinear Sci. Numer. Simulat. 2013. 18 (11): 3061-3074.

[19] Qi, T. Liu, Y. and Zou, Y. Existence result for a class of coupled fractional differential systems with integral boundary value conditions. J. Nonlinear Sci. Appl. 2017. 10 (7): 4034-4045.

[20] Wang, Y. Liu, L. and Wu, Y. Positive solutions for a class of fractional boundary value problem with changing sign nonlinearity. Nonlinear Anal. 2011. 74 (17): 6434-6441.

[21] Zou, Y. and He, G. On the uniqueness of solutions for a class of fractional differential equations. Appl. Math. Lett. 2017. 74: 68-73.

[22] Cui, Y. Ma, W. Sun, Q. and Su, X. New uniqueness results for boundary value problem of fractional differential equation. Nonlinear Analysis: Modelling and Control. 2018. 23(1): $31-39$.

[23] Bai, Z. Zhang, S. Sun, S. and Yin C. Monotone iterative method for fractional differential equations. Electron. J. Differential Equations 2016. 2016 (6): 1-8.

[24] Mawhin, J. and Willem, M. Critical Point Theorey and Hamiltonian Systems. Springer, New York, 1989.

[25] Rabinowitz, P.H. Minimax Methods in Critical Point Theory with Applications to Differential Equations. In: CBMS, vol. 65, Amer. Math. Soc. 1986. 
[26] Struwe, M. Variational Methods: Applications to Nonlinear Partial Differential Equations and Hamiltonian Systems. Ergebnisse der Mathematik und ihrer Grenzgebiete, 3, SpringerVerlag, Berlin-Heidelberg, 1990.

[27] Willem, M. Minimax Theorems. Birkhäuser, 1996.

[28] Jiao, F. and Zhou, Y. Existence of solutions for a class of fractional boundary value problems via critical point theory. Comput. Math. Appl. 62 (2011), 1181-1199.

[29] Ferrara M., and Hadjian, A. Variational approach to fractional boundary value problems with two control parameters. Electron. J. Differential Equations 2015. 2015 (138): 1-15.

[30] Heidarkhani, S. Multiple solutions for a nonlinear perturbed fractional boundary value problem. Dynam. Systems Appl. 2014. 23: 317-332.

[31] Zhao, Y. Chen, H. and Qin, B. Multiple solutions for a coupled system of nonlinear fractional differential equations via variational methods. Appl. Math. Comput. 2015. 257: 417427.

[32] Bonanno, G. and Marano, S.A. On the structure of the critical set of non-differentiable functions with a weak compactness condition. Appl. Anal. 2010. 89: 1-10.

[33] Afrouzi, G.A. Hadjian, A. and Heidarkhani, S. Multiplicity results for a class of two-point boundary value systems investigated via variational methods. Bull. Math. Soc. Sci. Math. 2012. 55 (103): 343-352.

[34] Bonanno, G. and Chinnì, A. Existence of three solutions for a perturbed two-point boundary value problem. Appl. Math. Lett. 2010. 23 (7): 807-811.

[35] Bonanno, G. and D'Aguì, G. Multiplicity results for a perturbed elliptic Neumann problem. Abstr. Appl. Anal. 2010. Article ID 564363, 10 pages.

[36] Candito, P. and D'Aguì, G. Three solutions to a perturbed nonlinear discrete Dirichlet problem. J. Math. Anal. Appl. 2011. 375 (2): 594-601.

[37] Heidarkhani, S. and Henderson, J. Critical point approaches to quasilinear second order differential equations depending on a parameter. Topol. Methods Nonlinear Anal. 2014. 44 (1): 177-197.

[38] Heidarkhani, S. and Henderson, J. Multiple solutions for a nonlocal perturbed elliptic problem of p-Kirchhoff type. Comm. Appl. Nonlinear Anal. 2012. 19 (3): 25-39.

[39] Zhao, Y. Chen, H. and Zhang, Q. Infinitely many solutions for fractional differential system via variational method. J. Appl. Math. Comput. 2016. 50 (1-2): 589-609.

[40] Bai, C. Existence of solutions for a nonlinear fractional boundary value problem via a local minimum theorem. Electron. J. Differential Equations 2012. 2012 (176): 1-9. 